\title{
The effectiveness of commonly used mouthwashes for the prevention of chemotherapy-induced oral mucositis: a systematic review
}

\author{
C.M.J. POTTING, MSC, RN, NURSE SCIENTIST Department of Haematology, Radboud University Nijmegen Medical \\ Centre, R. UITTERHOEVE, MSN, RN, NURSE sCientist Department of Medical Oncology, Radboud University \\ Nijmegen Medical Centre, W. SCHOLTE OP REIMER, PHD, RN, EPIDEMIOLOGIST Department of Cardiology, Eras- \\ mus Medical Centre Rotterdam, \& T. VAN ACHTERBERG, PHD, RN, Professor Centre for Quality of Care \\ Research, Department of Nursing Science, Radboud University Nijmegen Medical Center, the Netherlands
}

POTTING C.M.J., UITTERHOEVE R., SCHOLTE OP REIMER W. \& VAN ACHTERBERG T. (2006) European Journal of Cancer Care 15, 431-439

The effectiveness of commonly used mouthwashes for the prevention of chemotherapy-induced oral mucositis: a systematic review

Daily chlorhexidine mouthwash is often recommended for preventing chemotherapy-induced oral mucositis. Povidone-iodine, $\mathrm{NaCl} 0.9 \%$, water salt soda solution and chamomile mouthwash are also recommended. However, the effectiveness of these mouthwashes is unclear. Therefore, we performed a systematic review to assess the effectiveness of mouthwashes in preventing and ameliorating chemotherapy-induced oral mucositis. Based on study quality, three out of five randomized controlled trials were included in a meta-analysis. The results failed to detect any beneficial effects of chlorhexidine as compared with sterile water, or $\mathrm{NaCl} 0.9 \%$. Patients complained about negative side-effects of chlorhexidine, including teeth discoloration and alteration of taste in two of the five studies on chlorhexidine. The severity of oral mucositis was shown to be reduced by $30 \%$ using a povidone-iodine mouthwash as compared with sterile water in a single randomized controlled trial. These results do not support the use of chlorhexidine mouthwash to prevent oral mucositis.

Keywords: oral mucositis, chemotherapy, mouthwashes, chlorhexidine, oral care.

\section{INTRODUCTION}

Oral mucositis occurs in about $40 \%$ of patients who undergo cytostatic chemotherapy for malignancies (Scully \& Epstein 1996; Sonis et al. 2004). Virtually every patient who has undergone myeloablative therapy to prepare for a haematopoietic stem cell transplant (HSCT) develops mucositis, with $67 \%$ developing severe oral mucositis (Wardley et al. 2000).

Correspondence address: Carin Potting, Radboud University Nijmegen, Medical Centre, P/A Haematology 492, PO Box 9101, 6500 HB Nijmegen, the Netherlands (e-mail: c.potting@cis.umcn.nl).

Accepted 24 February 2006

DOI: $10.1111 / j .1365-2354.2006 .00684 . x$

European Journal of Cancer Care, 2006, 15, 431-439
Damage to the mucous membranes (mucositis) can occur as a consequence of the direct effects of cytostatic drugs on the rapidly dividing cells in the tissues in the mouth. The initial symptoms of mucositis usually present between the fourth and seventh day after chemotherapy (Wojtaszek 2000). White discoloration of the mucous membranes mostly precedes the redness, oedema and lesions. These lesions can develop into large painful ulcers that can seriously hinder eating and drinking (Rogers 2001). Furthermore, the protective effect of saliva can be reduced - due to a decrease in the quality and quantity increasing the chance of developing infection (Carl \& Havens 2000; Epstein et al. 2002).

Severe mucositis results in a significant reduction in the quality of life, potential nutritional deficit and even the 
postponement of chemotherapy (Bolwell et al. 2002). A recent study among 92 stem cell transplant recipients in eight centres in the United States, Canada and Europe demonstrated that the amount and severity of oral mucositis correlated with the number of days that patients required intravenous antibiotics, analgesics and parenteral feeding. The severity of mucositis among stem cell transplant recipients was also correlated with the number of admissions and readmissions, hospital costs and mortality (Sonis et al. 2001).

The high incidence and severe consequences of mucositis among patients who undergo chemotherapy underline the importance of good prevention.

Rinsing the mouth daily with chlorhexidine solution is a preventive measure frequently recommended by nurses. Solutions of sodium bicarbonate, chamomile and $0.9 \%$ saline are also often used in the Netherlands (Nieweg et al. 1992). The extent to which these mouthwashes actually help to prevent mucositis is unclear. Clinical practice guidelines for the prevention and treatment of cancer therapy-induced oral and gastrointestinal mucositis have been produced (Rubenstein et al. 2004), but only two studies were used as evidence to support the use of chlorhexidine although there are more studies available in the international literature. Moreover, there was no metaanalysis. There is also a review by Clarkson et al. (2003) involving patients who received chemotherapy and/or radiotherapy. However, it is commonly known that mucositis induced by chemotherapy differs from that induced by radiation (Rubenstein et al. 2004). Therefore, we undertook to search the international literature afresh to ascertain whether these mouthwashes actually contribute to the prevention of oral mucositis among patients who undergo treatment with cytostatic chemotherapy.

\section{METHOD}

\section{Search strategy}

The Medline and Cinahl databases were searched for the relevant literature published from 1992 to the autumn of 2004. The search was restricted to these years in order to obtain maximal validity in the light of oncology care today. The search terms 'mucositis, 'stomatitis' and 'chemotherapy' were used in combination with 'prevention', 'mouthwashes', 'antiseptic', 'oral infection', 'chlorhexidine', 'chamomile', 'PVP-iodine' and 'sodium bicarbonate'.

\section{Selection criteria}

All randomized studies of the effect of mouthwashes for the prevention and amelioration of oral mucositis in adult patients undergoing chemotherapy were eligible for this systematic literature study. Two independent assessors C. P. en R. U. selected the articles. The titles and/or abstracts were used to identify those that meet the inclusion criteria. Studies were selected if they involved using mouthwashes for oral mucositis, had a controlled study design, involved adult patients with cancer who received chemotherapy and included an outcome measure of the severity of mucositis. If a difference of opinion arose, a third author was consulted before the article was included or excluded.

\section{Study quality and analysis}

The quality of a systematic review is related to the quality of the studies used with randomized controlled trials topping the hierarchy of evidence (Juni et al. 2001). Quality assessment allows appraisal of the studies included and also aids data synthesis. The quality of studies was assessed for randomization, blinding and the intention-totreat analysis. In randomized controlled trials, patients are randomly assigned to either control or an experimental group. For blinding, a trial was classified as adequate if it was described as 'double-blind', a type of clinical trial in which neither the subject nor the investigator knows what treatment the patient is receiving. An intention-totreat analysis specifies how to handle non-compliant patients in a randomized controlled trial. This analysis requires that patients be analysed in the groups into which they were randomized, regardless of whether or not they complied with the treatment allocated (HuwilerMuntener et al. 2002).

In a meta-analysis or statistical pooling, the data gathered in the framework of a systematic review are statistically combined to estimate the effect of the intervention studied in the research (Thompson 1994).

The findings of the individual studies were analysed in a meta-analysis using the software Review Manager 4.2 (The Cochrane Collaboration 2002). In systematic reviews, homogeneity refers to the degree to which the results of studies included in a review are similar. A fixed effect model was allowed since homogeneity was found between the studies (Chi-squared test: $P<0.1$ ).

This is a statistical model that stipulates that the units under analysis (people in a trial or study in a meta-analysis) are the ones of interest, and thus constitutes the entire population of units. Only within-study variation is taken to influence the uncertainty of results (as reflected in the confidence interval) of a meta-analysis using a fixed effect model. Variation between the estimates of effect from each study (heterogeneity) does not effect the confidence interval in a fixed effect model. 


\section{RESULTS}

The search term 'mucositis or stomatitis' provided 7589 hits using Medline and Cinahl for the period 1992-2004. When combined with 'prevention', there were still 905 articles. After combining with 'mouthwashes', 'antiseptic', 'oral infection', 'chlorhexidine', 'chamomile', 'PVPiodine' or 'sodium bicarbonate', 20 articles remained (Fig. 1). Five of these studies investigated chlorhexidine in a randomized controlled clinical trial (RCT) (Epstein et al. 1992; Rutkauskas \& Davis 1993; Dodd et al. 1996, 2000; Pitten et al. 2003). Three articles were found which investigated iodine solution as a mouthwash. However, further investigation revealed that these three articles were all reports of the same study. Hence only the most complete one is included in the review (Adamietz et al. 1998).

One study determined the effects of chamomile solution (Fidler et al. 1996). The other 11 articles were excluded: five were not RCTs but tutor reviews, two investigated dental problems and two discussed guidelines for mucositis and were therefore excluded. One study investigated micronized sucralfate versus salt and soda mouthwashes in head and neck cancer patients who received radiation therapy. This study was excluded because it dealt with radiation-induced mucositis and not chemotherapy-induced mucositis. Another study also investigated sodium bicarbonate, but did not use a ran-

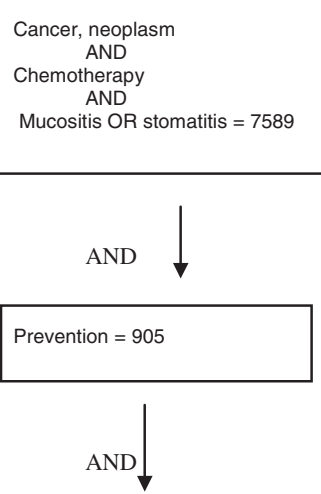

Mouthwashes or chlorhexidine OR chamomile OR PVP-iodine or sodium bicarbonate $=20$

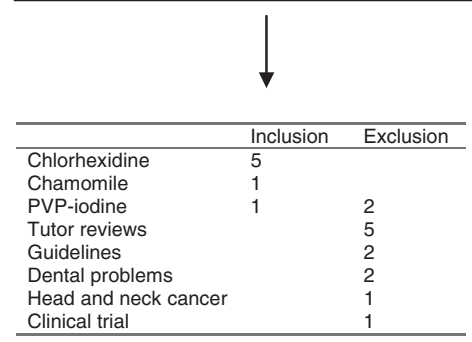

Figure 1. Selection of articles. domized study design (clinical trial) and was therefore excluded.

No RCTs investigating sodium bicarbonate were found. However, three articles were found which investigated salt and soda, this solution is similar to sodium bicarbonate. In one study, where chlorhexidine was used as the intervention, the control group used a water, salt and soda solution. This study was included (Dodd et al. 2000).

\section{Study characteristics}

The seven studies (Tables 1 and 2) included data from 863 adults with cancer with a mean age of $53.6,72 \%$ of the patients in the studies received chemotherapy, only $6 \%$ of the patients received HSCT, for $22 \%$ of the patients it is unknown which treatment was received.

The World Health Organization instrument (Miller et al. 1981) was used to score mucositis in three studies (Fidler et al. 1996; Adamietz et al. 1998; Pitten et al. 2003): one study adapted this scale (Rutkauskas \& Davis 1993), two studies (Dodd et al. 1996, 2000) used the Oral Assessment Guide (Eilers et al. 1988) and one study employed a four-point scale developed by the investigator (Epstein et al. 1992). The frequency of assessing mucositis varied from once to twice a day, once weekly, and on three separate occasions during treatment.

\section{Study quality}

All studies randomly allocated subjects to either an intervention or a comparison group. Only one study assigned patients to one of the treatment groups by stratified block randomization (Pitten et al. 2003), the blocks being selected using a set of random sampling numbers. A double-blind study design was used in five studies, though the group assignment was revealed in one study at an early stage (Rutkauskas \& Davis 1993). Another study had an open study design (Adamietz et al. 1998) and the last study did not report blinding at all (Epstein et al. 1992). The analysis was conducted on an intention-to-treat basis in four studies (Epstein et al. 1992; Fidler et al. 1996; Adamietz et al. 1998; Pitten et al. 2003).

\section{Compliance}

The compliance of patients with treatment has an important effect on the results of different studies, and is therefore an important element to consider (Boudes 1998). However, patient compliance was assessed in only three studies. Dodd et al. (2000) collected the mouth rinse bottles and measured the amounts remaining and 


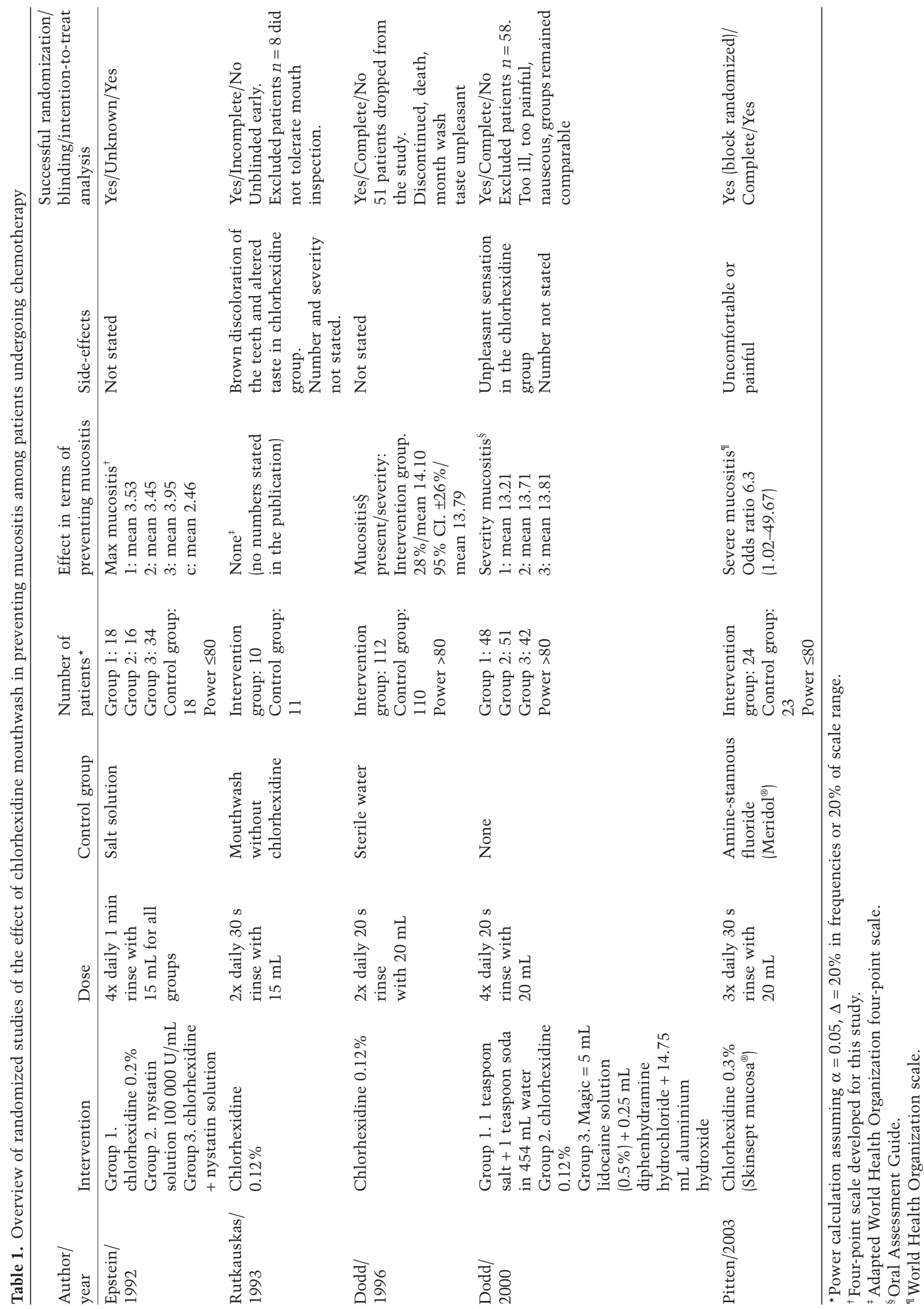




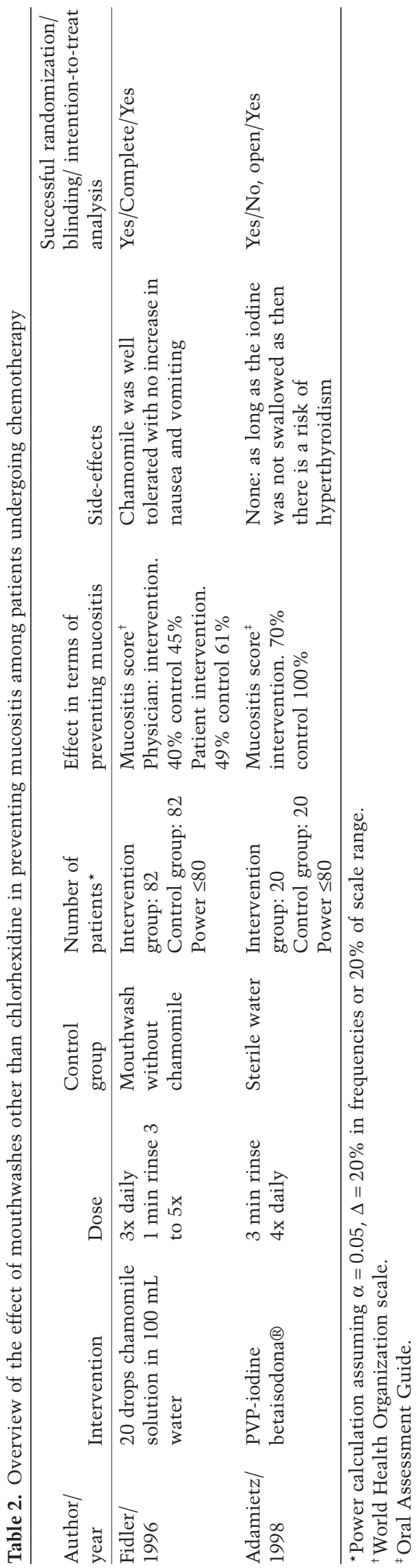

compared this with what should have been used. Compliance in this study was very high $(92 \%)$, although it is not known if patients disposed of their mouth rinses in another manner, but the investigators had no reason to believe that this occurred. However, Epstein et al. (1992) found less positive results regarding compliance with rinsing. In their study, assessment of compliance was based on medication records and on an interview at weekly assessment visits. Patients assigned to rinsing with Nystatin alone or in combination with chlorhexidine showed poor compliance, with only $47 \%$ of patients in the nystatin-chlorhexidine rinse group using the rinse a $100 \%$ of the time, $78 \%$ of patients using chlorhexidine at all times, but $89 \%$ of them using the saline solution group at all times.

Pitten et al. (2003) used brown glass bottles. On visiting the patients to assess mucositis, the clinician checked if the volume remaining in the bottle correlated with the number of rinses. The findings indicated that the patients had rinsed properly.

\section{Chlorhexidine}

Chlorhexidine is approved for use as an antibacterial mouthwash at a concentration of $0.12 \%$ and $0.2 \%$ to prevent the build-up of dental plaque and to prevent gingivitis (Yates et al. 2002). Its broad spectrum of antibacterial activity, minimal systemic absorption and ability to bind to oral surfaces led to use as prophylaxis in an attempt to prevent the development of oral mucositis (Matthijs \& Adriaens 2002).

Chlorhexidine has been tested in five randomized studies for its effects in preventing oral mucositis in patients undergoing chemotherapy Table 1.

Epstein et al. (1992) investigated three different mouthwashes, chlorhexidine, nystatin and a combination of nystatin-chlorhexidine and compared these with rinsing using a saline solution. All patients $(n=86)$ who received medical therapy that resulted in severe neutropenia were included into the study. Fifty-six patients $(65 \%)$ received aggressive chemotherapy for remission induction or consolidation. Thirty patients (35\%) received HSCT. The patients were asked to rinse with the mouthwash after eating. Oral hygiene was assessed using the gingival index and plaque levels, and mucositis was assessed using a four-point scale specially developed for the study. Bacterial and fungal oral cultures were done on a weekly basis. There was no difference in mucositis between the four groups although bacterial and fungal infections were found less often among the patients using chlorhexidine. 
Rutkauskas and Davis (1993) investigated the effect of chlorhexidine versus a placebo in patients undergoing HSCT or remission-indication chemotherapy. The study showed chlorhexidine to be ineffective in preventing mucositis. Unfortunately, the data were also presented in a form that made it impossible to include them in the meta-analysis.

Dodd et al. (1996) investigated the effect of an instruction programme for the systematic oral care of 222 patients undergoing chemotherapy provided by nurses in combination with two mouthwashes (chlorhexidine and sterile water). The preventative effects of rinsing with chlorhexidine were no greater than those of rinsing with sterile water leading the investigators to recommended rinsing with water only.

Dodd et al. (2000) also compared the preventative effects of three mouthwashes (chlorhexidine, salt and soda in water) and 'magic mouthwash' (containing Lidocaine, Benadryl and Maalox) in patients who received stomatotoxic chemotherapy at home and were monitored on an outpatient basis. Nurses used the Oral Assessment Guide for initial assessment, instructed patients on how to assess their own mouths, then phoned the patients every other day to note their oral status. No differences in the severity of mucositis were found between the three groups nor were there any significant differences in the time taken for signs and symptoms of mucositis to subside. The first signs of mucositis were seen within 6.6 days in the chlorhexidine group, within 7.0 days in the water/salt/ soda group and within 7.2 days in the 'magic mouthwash' group.

Pitten et al. (2003) investigated chlorhexidine versus amine-stannous fluoride solution to investigate whether leucopenic patients who cannot clean their teeth mechanically might have clinical benefit from an antiseptic mouth rinse containing chlorhexidine. The statistical analysis showed that there was a significant decrease in the numbers of microorganisms in the oral cavity during leucopenia among those in the chlorhexidine group compared with that in the control group. However, this did not translate into any measurable clinical benefit. Patients rinsing with chlorhexidine also indicated that the rinsing was unpleasant and even painful.

\section{Power}

None of the studies reported a power calculation, so we calculated this from the numbers of patients reported, assuming an alpha of 0.05 (two-sided) and a clinically relevant effect size of $20 \%$ of the scale range for severity of mucositis in several studies, or a $20 \%$ difference in frequencies in studies with presence or absence of mucositis as the main outcome. Only the studies by Dodd et al. $(1996,2000)$ had sufficient power $(\geq 80 \%)$. The study by Fidlet et al. had an estimated power of 70\% (Fidler et al. 1996), whereas the power of the other studies was less.

\section{Meta-analysis}

Within the meta-analysis, the results from individual studies were weighted in inverse proportion to their variance, resulting in a weight proprtional to the size of the studies.

Four out of five studies that investigated chlorhexidine mouthwash for preventing mucositis were eligible for inclusion in the meta-analysis. Rutkauskas and Davis (1993) and Pitten et al. (2003) did not state all of the necessary figures for this. The study by Epstein et al. (1992) had a total of four groups and in the analysis was approached as two studies, namely chlorhexidine versus saline solution and chlorhexidine + nystatin versus nystatin.

The study by Dodd et al. (2000) was also entered as two studies (chlorhexidine vs. water/salt and soda and chlorhexidine vs. 'magic mouthwash').

All the information is contained within the forest plot graphical representation of the results in Figure 2.

Taken together the results, the five studies showed no significant effect of chlorhexidine mouthwash (Weighted mean differences $0.22 ; 95 \%$ confidence interval $=-0.20$, $0.63)$. The test for heterogeneity and the test for overall effect are given at the bottom of the forest plot. It is important to remember that heterogeneity may be present when all or most studies indicate the same treatment effect, but the size of the effect differs or the trials are contradictory about the effect (Sutton et al. 1998). The results are considered homogenous when the effect sizes differ due to sampling errors.

\section{Other mouthwashes}

Table 2 provides an overview of randomized studies into the effect of mouthwashes other than chlorhexidine in preventing mucositis in patients undergoing chemotherapy.

Fidler et al. (1996) evaluated the effect of a chamomile solution in a group with a total of 164 patients treated with 5-FU chemotherapy. After randomization, 82 patients received a mouthwash with a chamomile solution and 82 patients received a mouthwash without. All patients received oral cryotherapy for $30 \mathrm{~min}$ with each 


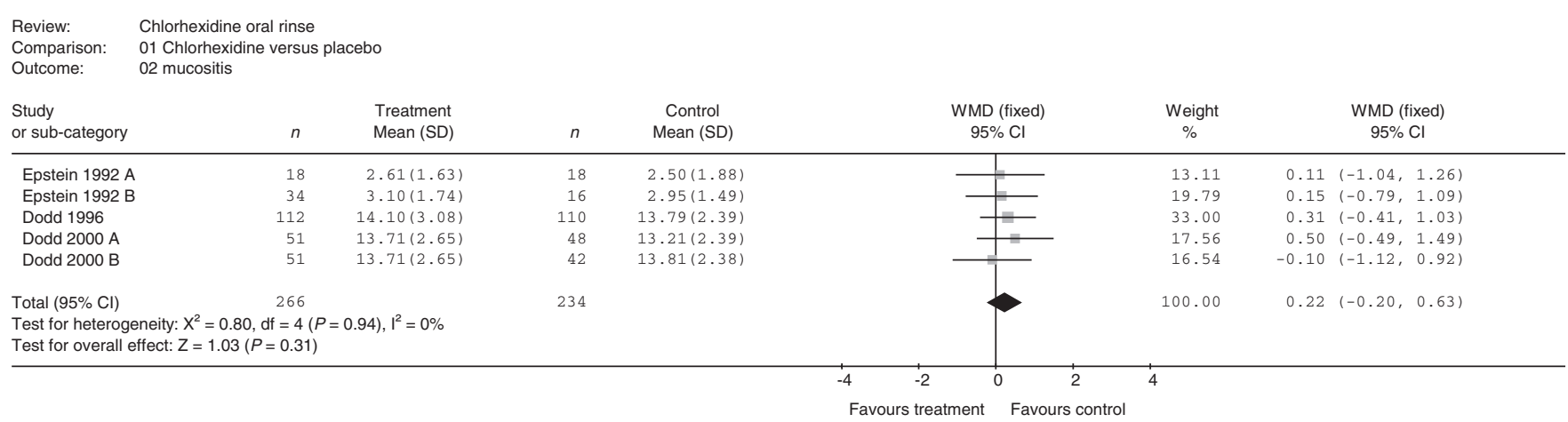

Figure 2. Forest plot of mean (SD) mucositis scores. CI, confidence interval; SD, standard deviation; WMD, weighted mean difference.

dose of 5-FU. Mucositis was scored by the physician (scale of $0-4)$. The patient also recorded his or her score on a daily basis. No differences were found between the chamomile group and the control group in either the incidence or severity of mucositis.

Adamietz et al. (1998) investigated the preventative effects of iodine solution as a mouthwash compared with rinsing with water in 40 patients given radiochemotherapy ( $n=20$ for both groups). The World Health Organization criteria for mucositis (scale of $0-4$ ) were used to estimate the severity and duration of the mucositis. The iodine group had a significantly less severe mucositis compared with the control group and the duration of the mucositis was shorter (2.8 weeks for the iodine group vs. 9.3 weeks for the control group). However, the study was too small to be confident that the difference observed was not simply a chance finding.

\section{CONCLUSION}

A systematic review was used to assemble and synthesize the evidence for the effect of commonly used mouthwashes on the prevention of chemotherapy-induced oral mucositis. Comprehensive search methods were used to minimize any bias.

With the exception of iodine solution, none of the studies investigated were able to demonstrate an effect in preventing mucositis in patients undergoing chemotherapy.

\section{Chlorhexidine}

Chlorhexidine is widely used and has been investigated albeit in various small studies. Individually, the studies found chlorhexidine to be ineffective and increasing power through meta-analysis did not alter this. Studies done before 1992 found a positive effect of rinsing with chlorhexidine (McGaw \& Belch 1985; Ferretti et al. 1988, 1990) whereas those conducted in the period 1992-2004 found either no effect or a negative effect. One possible explanation could be that bacterial infections were better controlled and managed after 1992 than before because of better antibiotics.

The discoloration of teeth, the bitter taste and the unpleasant sensation experienced together with ineffectiveness are sufficient reasons for recommending sterile water, $0.9 \%$ saline solution or sodium bicarbonate (water, salt and soda) rather than chlorhexidine. Furthermore, these alternatives are less expensive and readily available in everyday nursing practice.

\section{Other mouthwashes}

Most of the other formulations had no effect on the prevention of mucositis. The antifungal drug nystatin, even in combination with chlorhexidine, was no exception (Epstein et al. 1992).

Even chamomile, which has an anti-inflammatory effect, proved ineffective. (Carl \& Emrich 1991).

One study did demonstrate that iodine solution was effective as a mouthwash, but this finding must be treated with caution, due to the small sample sizes involved. Moreover, side-effects were not reported, though, when accidentally swallowed, iodine can cause hyperthyroidism.

The sample sizes varied from 21 to 222 and none of the studies indicated the power calculation based on a proposed treatment effect even though adequate statistical power is crucial to minimize type-II or beta errors (Cohen 1992). This shortcoming was compensated to some extent by the meta-analysis supporting the negative conclusions for chlorhexidine, which does not apply to other mouthwashes.

Patient compliance with the intervention has an important effect on the results and should always be considered (Boudes 1998), yet only three studies did so (Epstein et al. 1992; Dodd et al. 2000, Pitten et al. 2003). 
Based on our findings and those of others (Clarkson et al. 2003; Rubenstein et al. 2004), the use of chlorhexidine as well as other mouthwash for preventing oral mucositis in patients undergoing chemotherapy cannot be recommended. The use of an iodine solution could be promising, but should be investigated further.

\section{ACKNOWLEDGEMENTS}

This literature study was part of the State of the Art study which was being carried out within the framework of the research programme 'Tussen weten en doen' [Between knowledge and practice] funded by the Netherlands Organization for Health Research and Development (Dutch acronym: ZonMw) and coordinated by the Netherlands Centre for Excellence in Nursing (Dutch acronym: LEVV).

\section{REFERENCES}

Adamietz I.A., Rahn R., Bottcher H.D., Schafer V., Reimer K. \& Fleischer W. (1998) Prophylaxis with povidone-iodine against induction of oral mucositis by radiochemotherapy. Supportive Care in Cancer 6, 373-377.

Bolwell B.J., Kalaycio M., Sobecks R., Andresen S., Kuczkowski E., Bernhard L., Lomax R., Kohuth J., Mendiola S., Rybicki L. \& Pohlman B. (2002) A multivariable analysis of factors influencing mucositis after autologous progenitor cell transplantation. Bone Marrow Transplantation 30, 587-591.

Boudes P. (1998) Drug compliance in therapeutic trials: a review. Controlled Clinical Trials 19, 257-268.

Carl W. \& Emrich L.S. (1991) Management of oral mucositis during local radiation and systemic chemotherapy: a study of 98 patients. Journal of Prosthetic Dentistry 66, 361-369.

Carl W. \& Havens J. (2000) The cancer patient with severe mucositis. Current Review of Pain 4, 197-202.

Clarkson J.E., Worthington H.V. \& Eden O.B. (2003) Interventions for preventing oral mucositis for patients with cancer receiving treatment. Cochrane Database of Systematic Reviews CD000978.

Cohen J.A. (1992) A power primer. Psychological Bulletin 12, 155-159.

Dodd M.J., Larson P.J., Dibble S.L., Miaskowski C., Greenspan D., MacPhail L., Hauck W.W., Paul S.M., Ignoffo R. \& Shiba G. (1996) Randomized clinical trial of chlorhexidine versus placebo for prevention of oral mucositis in patients receiving chemotherapy. Oncology Nursing Forum 23, 921-927.

Dodd M.J., Dibble S.L., Miaskowski C., MacPhail L., Greenspan D., Paul S.M., Shiba G. \& Larson P. (2000) Randomized clinical trial of the effectiveness of 3 commonly used mouthwashes to treat chemotherapy-induced mucositis. Oral Surgery Oral Medicine Oral Pathology Oral Radiology and Endodontics 90, 39-47.

Eilers J., Berger A.M. \& Petersen M.C. (1988) Development, testing, and application of the oral assessment guide. Oncology Nursing Forum 15, 325-330.

Epstein J.B., Vickars L., Spinelli J. \& Reece D. (1992) Efficacy of chlorhexidine and nystatin rinses in prevention of oral complications in leukemia and bone marrow transplantation. Oral Surgery, Oral Medicine, Oral Pathology 73, 682689.
Epstein J.B., Tsang A.H., Warkentin D. \& Ship J.A. (2002) The role of salivary function in modulating chemotherapy-induced oropharyngeal mucositis: a review of the literature. Oral Surgery, Oral Medicine, Oral Pathology, Oral Radiology and Endodontics 94, 39-44.

Ferretti G.A., Ash R.C., Brown A.T., Parr M.D., Romond E.H. \& Lillich T.T. (1988) Control of oral mucositis and candidiasis in marrow transplantation: a prospective, double-blind trial of chlorhexidine digluconate oral rinse. Bone Marrow Transplantation 3, 483-493.

Ferretti G.A., Raybould T.P., Brown A.T., Macdonald J.S., Greenwood M., Maruyama Y., Geil J., Lillich T.T. \& Ash R.C. (1990) Chlorhexidine prophylaxis for chemotherapy- and radiotherapy-induced stomatitis: a randomized double-blind trial. Oral Surgery, Oral Medicine, Oral Pathology 69, 331-338.

Fidler P., Loprinzi C.L., O'Fallon J.R., Leitch J.M., Lee J.K., Hayes D.L., Novotny P., Clemens-Schutjer D., Bartel J. \& Michalak J.C. (1996) Prospective evaluation of a chamomile mouthwash for prevention of 5-FU-induced oral mucositis. Cancer 77, 522525 .

Huwiler-Muntener K., Juni P., Junker C. \& Egger M. (2002) Quality of reporting of randomized trials as a measure of methodologic quality. Journal of the American Medical Association 287, 2801-2804.

Juni P., Altman D.G. \& Egger M. (2001) Systematic reviews in health care: assessing the quality of controlled clinical trials. British Medical Journal 323, 42-46.

McGaw W.T. \& Belch A. (1985) Oral complications of acute leukemia: prophylactic impact of a chlorhexidine mouth rinse regimen. Oral Surgery, Oral Medicine, Oral Pathology 60, 275280.

Matthijs S. \& Adriaens P.A. (2002) Chlorhexidine varnishes: a review. Journal of Clinical Periodontology 29, 1-8.

Miller A.B., Hoogstraten B., Staquet M. \& Winkler A. (1981) Reporting results of cancer treatment. Cancer 47, 207-214.

Nieweg R., van Tinteren H., Poelhuis E.K. \& Abraham-Inpijn L. (1992) Nursing care for oral complications associated with chemotherapy. A survey among members of the Dutch Oncology Nursing Society. Cancer Nursing 15, 313-321.

Pitten F., Kiefer T., Buth C., Doelken G. \& Kramer A. (2003) Do cancer patients with chemotherapy-induced leukopenia benefit from an antiseptic chlorhexidine-based oral rinse? A doubleblind, block-randomized, controlled study. Journal of Hospital Infection 53, 283-291.

Rogers B.B. (2001) Mucositis in the oncology patient. Nursing Clinics of North America 36, 745-760.

Rubenstein E.B., Peterson D.E., Schubert M., Keefe D., McGuire D., Epstein J., Elting L.S., Fox P.C., Cooksley C. \& Sonis S.T. (2004) Clinical practice guidelines for the prevention and treatment of cancer therapy-induced oral and gastrointestinal mucositis. Cancer 100, 2026-2046.

Rutkauskas J.S. \& Davis J.W. (1993) Effects of chlorhexidine during immunosuppressive chemotherapy. A preliminary report. Oral Surgery, Oral Medicine, Oral Pathology 76, 441-448.

Scully C. \& Epstein J.B. (1996) Oral health care for the cancer patient. European Journal of Cancer B Oral Oncology 32B, 281-292.

Sonis S.T., Oster G., Fuchs H., Bellm L., Bradford W.Z., Edelsberg J., Hayden V., Eilers J., Epstein J.B., Leveque F.G., Miller C., Peterson D.E., Schubert M.M., Spijkervet F.K. \& Horowitz M. (2001) Oral mucositis and the clinical and economic outcomes of hematopoietic stem-cell transplantation. Journal of Clinical Oncology 19, 2201-2205.

Sonis S.T., Elting L.S., Keefe D., Peterson D.E., Schubert M., Hauer-Jensen M., Bekele B.N., Raber-Durlacher J., Donnelly 
J.P. \& Rubenstein E.B. (2004) Perspectives on cancer therapyinduced mucosal injury: pathogenesis, measurement, epidemiology, and consequences for patients. Cancer 100, 1995-2025. Sutton A.J., Abrams K.R., Jones D.R., Sheldon T.A. \& Song F. (1998) Systematic reviews of trials and other studies. Health Technology Assessment 2, 1-276.

Thompson S.G. (1994) Systematic review: why sources of heterogeneity in meta-analysis should be investigated. British Medical Journal 309, 1351-1355.

Wardley A.M., Jayson G.C., Swindell R., Morgenstern G.R.,
Chang J., Bloor R., Fraser C.J. \& Scarffe J.H. (2000) Prospective evaluation of oral mucositis in patients receiving myeloablative conditioning regimens and haemopoietic progenitor rescue. British Journal of Haematology 110, 292-299.

Wojtaszek C. (2000) Management of chemotherapy-induced stomatitis. Clinical Journal of Oncology Nursing 4, 263-270.

Yates R., Shearer B.H., Huntington E. \& Addy M. (2002) A method to compare four mouthrinses: time to gingivitis level as the primary outcome variable. Journal of Clinical Periodontology 29, 519-523. 